\title{
Leucine Imparts Cardioprotective Effects by Enhancing mTOR Activity and Mitochondrial Fusion in a Myocardial Ischemia/reperfusion Injury Murine Model
}

\author{
Atsushi Morio \\ Rie Tsutsumi \\ University of Tokushima: Tokushima Daigaku \\ Shiho Satomi \\ Hiroshima University Hospital: Hiroshima Daigaku Byoin \\ Takashi Kondo \\ Hiroshima University Hospital: Hiroshima Daigaku Byoin \\ Hirotsugu Miyoshi \\ Hiroshima University Hospital: Hiroshima Daigaku Byoin

\section{Takahiro Kato} \\ Hiroshima University Hospital: Hiroshima Daigaku Byoin

\section{Masashi Kuroda} \\ University of Tokushima: Tokushima Daigaku

\section{Tadahiro Kitamura} \\ Gunma University: Gunma Daigaku

\section{Kenta Hara} \\ Kita Harima Medical Center: Kitaharima Sogo Iryo Center \\ Noboru Saeki \\ Hiroshima University Hospital: Hiroshima Daigaku Byoin

\section{Hiroshi Sakaue} \\ University of Tokushima: Tokushima Daigaku \\ Yasuo M. Tsutsumi ( $\nabla$ yasuo223@hiroshima-u.ac.jp ) \\ Hiroshima University Hospital: Hiroshima Daigaku Byoin
}

Hiroshima University Hospital: Hiroshima Daigaku Byoin https://orcid.org/0000-0001-9360-9437

\section{Research}

Keywords: Leucine, high-fat diet, myocardial ischemia/reperfusion injury, cardioprotective

Posted Date: May 5th, 2021 
DOl: https://doi.org/10.21203/rs.3.rs-475382/v1

License: (c) (1) This work is licensed under a Creative Commons Attribution 4.0 International License. Read Full License

Version of Record: A version of this preprint was published at Diabetology \& Metabolic Syndrome on November 20th, 2021. See the published version at https://doi.org/10.1186/s13098-021-00755-z. 


\section{Abstract}

Background: Coronary artery disease is a leading cause of morbidity and mortality among patients with diabetes. Previously, we demonstrated that branched-chain amino acids (BCAAs) showed cardioprotective effects against cardiac ischemia/reperfusion (I/R) injury. A recent study suggested that leucine (Leu), a BCAA, is a key amino acid involved in mammalian target of rapamycin (mTOR) activity and mitochondrial function. In this study, we examined the preconditioning effect of Leu treatment on diabetic hearts in mice.

Methods: In vivo mice models of I/R injury were divided into the following groups: control, $\mathrm{mTOR}^{+/-}$, and high-fat diet (HFD)-induced obese groups. Mice were randomly administered with Leu, the mTOR inhibitor rapamycin (Rap), or Leu with Rap. Isolated rat cardiomyocytes were subjected to simulated I/R injury. Biochemical and mitochondrial functional assays were performed to evaluate the changes in mTOR activity and mitochondrial dynamics caused by Leu treatment.

Results: Leu-treated mice showed a significant reduction in infarct size when compared with the control group ( $34.8 \% \pm 3.8 \%$ vs. $43.1 \% \pm 2.4 \%, p<0.05)$, whereas Rap-treated mice did not show the protective effects of Leu. This preconditioning effect of Leu was attenuated in $\mathrm{mTOR}^{+/-}$mice. Additionally, Leu promoted mitochondrial fusion in isolated cardiomyocytes. In HFD-induced obese mice, Leu treatment significantly reduced infarct size $(41.0 \% \pm 1.1 \%$ vs. $51.0 \% \pm 1.4 \%, p<0.05)$, which was not induced by ischemic preconditioning, and this effect was inhibited by Rap. Furthermore, we observed enhanced mTOR protein expression and mitochondrial fusion with decreased reactive oxygen species production.

Conclusions: Leu treatment improved the damage caused by myocardial I/R injury by promoting mTOR activity and mitochondrial fusion.

\section{Introduction}

Coronary artery disease (CAD) is a leading cause of morbidity and mortality among patients with diabetes, which increases the risk of developing CAD by 2- to 4-fold. [1] [2]Both type 1 and type 2 diabetic individuals are prone to developing ischemic heart disease, including acute myocardial infarction (AMI) and post-infarct complications. [3] Mortality from AMI is approximately twice among diabetic patients compared with that among non-diabetic individuals. [4] [5] Despite the burden of ischemic heart disease among patients with diabetes, effective treatment is currently unavailable. [6]

Myocardial ischemia/reperfusion (I/R) injury is a condition wherein the damage is caused by the occlusion of coronary arteries and restoration of blood flow to the ischemic myocardium. [7] [8] Several studies have reported the preconditioning effect of pharmacological agents through signaling pathways regulating cellular processes such as necrosis, apoptosis, and autophagy. [9] [10] [11]

Recently, we showed the cardiac preconditioning effect of branched-chain amino acid (BCAA) treatment on I/R injury in mice, resulting from increased mammalian target of rapamycin (mTOR) activity and 
improved mitochondrial function. [12] Among BCAAs, leucine (Leu) can activate mTOR kinase, thereby leading to the phosphorylation of p70S6 kinase and increasing the phosphorylation of serine residues in insulin receptor substrate-1 [13], which inhibits insulin signaling and insulin-stimulated glucose transport in muscles and fats. However, whether Leu has cardioprotective effects on diabetic hearts is unclear.

In this study, we examined the changes in mTOR activity and mitochondrial dynamics caused by Leu administered during I/R injury of the diabetic heart in a high-fat diet (HFD)-induced obese mouse model.

\section{Materials And Methods \\ 2.1. Animals}

All animals were treated in compliance with the guidelines for proper conduct of animal experiments and related activities (Ministry of Education, Culture, Sports, Science, and Technology of Japan). Additionally, the protocols, which follow the ARRIVE guidelines [14], were approved by the Animal Care and Use Committee at the University of Tokushima. Adult male Wistar rats and male C57BL/ 6 mice at 4 weeks of age were purchased from Japan SLC, Inc. (Shizuoka, Japan). $\mathrm{mTOR}^{+/-}$mice were used as previously described. [15] Mice were housed under temperature- $\left(23 \pm 3^{\circ} \mathrm{C}\right)$ and humidity-controlled conditions with a $12 \mathrm{~h}$ light/12 h dark cycle. Mice had free access to water and a control diet (14\% of calories from fat; Oriental Yeast Co., Ltd., Tokyo, Japan) or an HFD (60\% of calories from fat; Oriental Yeast Co., Ltd.). Mice were allowed to adapt to these conditions for 6 weeks before performing the experiments on them at 10 weeks of age.

\subsection{In vivo myocardial I/R experiments}

The surgical methods used were similar to those described previously. [16] [17] [18] Mice were anesthetized with sodium pentobarbital ( $80 \mathrm{mg} / \mathrm{kg}$, i.p.) and mechanically ventilated with $100 \%$ oxygen using a pressure-controlled ventilator (TOPO ventilator, Kent Scientific Co., Torrington, CT, USA). The core body temperature was maintained with a heating pad, and electrocardiogram leads were placed to record the heart rate. Ischemia was induced by occluding the left coronary artery (LAD) with a 7-0 silk suture for $30 \mathrm{~min}$, after which the ligature was released and the heart was perfused for $2 \mathrm{~h}$. Mice were randomly assigned to each experimental group. Saline $(0.9 \%)$ or Leu $(200 \mathrm{mg} / \mathrm{kg}$, IV) was administered $20 \mathrm{~min}$ before the occlusion. In the ischemic preconditioning (IPC) group, IPC was induced by occluding the LAD for $5 \mathrm{~min}$, followed by $15 \mathrm{~min}$ of reperfusion immediately before the I/R procedure.

\subsection{Western blotting}

Lysates were separated using sodium dodecyl sulfate-polyacrylamide gel electrophoresis on $10 \%$ polyacrylamide precast gels (Invitrogen, Carlsbad, CA, USA) and transferred to polyvinylidene difluoride membranes through electroelution. Membranes were blocked in $20 \mathrm{mM}$ Tris-buffered saline with $1 \%$ Tween containing $5 \%$ skimmed milk and incubated with primary antibodies overnight at $4{ }^{\circ} \mathrm{C}$. Immunolabeled blots were visualized using horseradish peroxidase-conjugated secondary antibodies 
(Santa Cruz Biotechnology, Santa Cruz, CA, USA) and enhanced chemiluminescence reagent (GE Healthcare, Waukesha, WI, USA). [19]

\subsection{Reactive oxygen species (ROS)}

To measure ROS production in the myocardium, the OxiSelect ${ }^{\mathrm{TM}}$ in vitro ROS/RNS Assay kit (Cell Biolabs, San Diego, CA, USA) was used. Before performing the I/R procedure, mice were injected with $200 \mu \mathrm{L}$ of saline, Leu, or Leu and rapamycin (Rap) into the aorta. All experimental procedures were performed according to the manufacturer's instructions. The ROS content was determined using the predetermined dichlorodihydrofluorescein standard curve, and mean fluorescence units were recorded.

\subsection{Isolation and maintenance of rat cardiomyocytes}

Cardiomyocytes were isolated from adult male Wistar rats. Rats were heparinized (1.0 IU/g, i.p.) 30 min before anesthetizing them with pentobarbital ( $80 \mathrm{mg} / \mathrm{kg}$, i.p.). Myocytes were obtained via enzymatic (210 U/mg collagenase II; Worthington, Lakewood, NJ, USA) digestion of the heart using a Langendorff apparatus. Enzymatic digestion was performed as previously described. [20] [21] Isolated myocytes were then cultured in $4 \%$ fetal bovine serum on laminin $\left(2 \mu \mathrm{g} / \mathrm{cm}^{2}\right)$-coated plates for $1 \mathrm{~h}$.

Culturing/maintenance media were changed to serum-free media [ $1 \%$ bovine serum albumin $+0.1 \%$ penicillin/streptomycin M199 media (Invitrogen, Carlsbad, CA, USA)] to eliminate all non-myocytes, and cardiac myocytes were incubated at $37^{\circ} \mathrm{C}$ in $5 \% \mathrm{CO}_{2}$ for $24 \mathrm{~h}$.

\subsection{Mitochondrial dynamics analysis in isolated rat cardiomyocytes}

Six hours prior to the pretreatment, all media were replaced with amino acid-free Dulbecco's Modified Eagle's medium to wash out any amino acids in M199 media. L-Leu (2.3 g; Sigma-Aldrich, St. Louis, MO, USA) was dissolved in $100 \mathrm{~mL}$ distilled water, creating a $175 \mathrm{mM}$ stock solution. Cells were pretreated with Leu $(160 \mu \mathrm{M})$ or control [phosphate-buffered saline (PBS)] for $2 \mathrm{~h}$. We evaluated the changes in mitochondrial dynamics of each group for $4 \mathrm{~h}$ at 30 min intervals after the administration of Leu or PBS.

After pretreatment with Leu or PBS, simulated ischemia/reperfusion was performed. Simulated ischemia was induced by replacing the air content with a $95 \% \mathrm{~N}_{2}$ and $5 \% \mathrm{CO}_{2}$ gas mixture at $2 \mathrm{~L} / \mathrm{min}$ in a chamber and the media with glucose-free media. This was performed for $60 \mathrm{~min}$, followed by $60 \mathrm{~min}$ of "reperfusion" by replacing the media with normal maintenance media and by incubating the cells with $21 \% \mathrm{O}_{2}$ and $5 \% \mathrm{CO}_{2}$. Finally, the cells were maintained in Krebs solution and fixed with $4 \%$ paraformaldehyde after incubating them for 30 min with MitoTracker Green FM (400 nmol/L; Molecular Probes, Invitrogen). Confocal image stacks were captured using a Leica laser microscope (Leica, Tokyo, Japan), as described previously. [22] Mitochondrial density was quantified using the ImageJ software $(\mathrm{NIH}$, Bethesda, MD, USA). The number and volume of each mitochondria were quantified using the Image J 3D Object Counter plug-in. The percentage of cells with a fusion pattern was determined based on the criteria that evaluated mitochondrial fusion with mitochondrial volume and a decrease in the number of mitochondria. [23] [24] 


\subsection{Electron microscopy}

Whole hearts or cardiomyocytes were fixed with $2.5 \%$ glutaraldehyde in $0.1 \mathrm{M}$ cacodylate buffer for $2 \mathrm{~h}$ at room temperature, post-fixed with $1 \%$ osmium tetroxide in $0.1 \mathrm{M}$ cacodylate buffer for $1 \mathrm{~h}$ at room temperature, and embedded as monolayers using LX-112 embedding kits (Ladd Research, Williston, VT, USA). Sections were stained with uranyl acetate and lead citrate, and observed under an electron microscope (EM). Random sections were taken by an EM technician who was blinded to the treatments. [25]

\subsection{Statistical Analyses}

All results were analyzed by observers who were blinded to the animal treatment history. Data are presented as mean \pm standard deviation. Differences between the treatment groups were tested for statistical significance by one-way analysis of variance, followed by Bonferroni's post hoc test. Differences were considered significant at $\mathrm{P}<0.05$.

\section{Results}

\subsection{Leu reduces infarct size in wild-type but not in $\mathrm{mTOR}^{+/-}$ mice}

The area at risk was calculated as a percentage of infarct size, and was found to be similar between the groups. In mice treated with Leu, a significant reduction in myocardial I/R injury was observed when compared with wild-type mice treated with vehicle control $(34.8 \% \pm 3.8 \%$ vs. $43.1 \% \pm 2.4 \%, n=7, p<0.05)$, but not in those treated with Leu and Rap. In addition, the effect of Leu was inhibited in $\mathrm{mTOR}^{+/-}$mice (Fig. 1).

\subsection{Leu promotes mitochondrial fusion in isolated rat cardiomyocytes}

In cardiomyocytes treated with Leu, mitochondria appeared to be interconnected. The percentage of cells displaying fused mitochondria was significantly increased at $2 \mathrm{~h}$ after Leu pretreatment when compared with the percentage of cells without Leu pretreatment $(20 \% \pm 8.5 \%$ vs. $78 \% \pm 6.2 \%, n=5, p<0.01)$

(Fig. 2a). The volume of individual mitochondria was measured using the three-dimensional reconstitution of confocal stacks. Leu caused a $71 \%$ increase in the volume of individual mitochondria at $2 \mathrm{~h}$ after Leu treatment (Fig. 2b). In addition, Leu stimulated mitochondrial fusion with an increase in mitochondrial size, whereas the number of mitochondria per cell was found to be decreased (Fig. $2 \mathrm{c}$ and 2d).

\subsection{Leu reduces infarct size in HFD-induced obese mice through mTOR activity}


In HFD-induced obese mice, IPC did not affect the infarct size caused by I/R injury. However, Leu treatment reduced the infarct size $(41.0 \pm 1.1 \%$ vs. $47.0 \pm 1.5 \%, n=7, p<0.05)$, whereas this effect was not observed in the group administered with Leu and Rap (Fig. 3a).

\subsection{Leu enhanced mTOR protein expression}

mTOR expression was enhanced by Leu administration in wild-type and HFD-induced obese mice, but not in $\mathrm{mTOR}^{+/-}$mice (Fig. 3b). However, Rap inhibited the increase in mTOR expression in wild-type and HFDinduced obese mice.

\subsection{Mitochondrial dynamics under in vivo conditions}

Mitochondrial dynamics were examined upon Leu administration in the heart tissue of HFD-induced obese mice (Fig. 3c) and we found that Leu administration enhanced mitochondrial fusion in the myocardium of HFD-induced obese mice.

\subsection{Leu decreased ROS production in wild-type and HFD- induced obese mice but not in $\mathrm{mTOR}^{+/-}$mice}

After the I/R procedure, ROS production was decreased by Leu treatment in wild-type and HFD-induced obese mice but not in $\mathrm{mTOR}^{+/-}$mice. Moreover, Rap inhibited the effect of Leu in wild-type and HFDinduced obese mice (Fig. 4).

\section{Discussion}

Through several experimental approaches, we have provided new evidence suggesting that Leu reduces the infarct size caused by myocardial I/R injury in wild-type and HFD-induced obese mouse models along with enhanced mitochondrial fusion. Leu did not affect infarct size in $\mathrm{mTOR}^{+/-}$mice, thereby suggesting that the effect of Leu was mediated through the mTOR signaling pathway. As observed in wild-type mice, Leu could impart protective effects on an HFD-induced obese mouse model by preconditioning treatment. Furthermore, Leu treatment led to an increase in mitochondrial fusion and a decrease in ROS production in diabetic hearts.

Leu is a BCAA that is important for protein synthesis. [26] Previously, we demonstrated that BCAAs show cardioprotective effects via the mTOR signaling pathway. [12] In this study, Leu reduced infarct size in wild-type mice, but not in $\mathrm{mTOR}^{+/-}$mice. Therefore, this result indicates that Leu is the key amino acid that stimulates mTOR activity and protects the heart from I/R injury.

Mitochondrial dynamics and coordinated fission and fusion cycles are important for maintaining the shape, distribution, and size of mitochondria. Mitochondria are involved in many cellular processes such as cell cycle, immunity, apoptosis, and mitochondrial quality control because of their transient and rapid morphological adaptability. [27] Disintegration of the reticular form of mitochondria into fragments has been considered as a physiological indicator of mitochondrial dysfunction. [28] Promoting fusion or 
inhibiting fragmentation of mitochondria prevents or delays the onset of apoptosis. [29] Several studies have shown that mTOR activity contributes to mitochondrial function. [30] Morita et al. demonstrated that mTOR complex 1 regulates mitochondrial dynamics and cell survival by stimulating mitochondrial fission process protein 1 translation. [31] Contrastingly, Szabo et al. showed that mitochondrial fusion with mTOR phosphorylation had a preventive effect on mitochondrial fragmentation. [32] Our results suggested that pharmacological preconditioning by treating myocardial I/R injury with Leu stimulates mitochondrial fusion. In addition, we found that mTOR activity contributes to mitochondrial fusion. Further studies are, however, needed to identify the detailed signaling pathways involved between mTOR activity and mitochondrial dynamics in cardiac preconditioning.

Recently, diabetes and insulin resistance have been shown to be associated with mitochondrial dysfunction. [33] [34] Cardiac preconditioning is attenuated by diabetes [35], and the loss of cardioprotective effects may be caused by the inhibition of insulin signaling. [3] However, the effects of Leu seem not to largely depend on the insulin-related mechanisms. [36] Previously, we demonstrated that the effects of BCAA preconditioning are mediated via the MTOR pathway and not the phosphoinositide 3kinase pathway, which is downstream of insulin receptor signaling. In this study, we demonstrated that Leu can recover the effect of preconditioning, which was attenuated in case of IPC, through mTOR activity in the diabetic heart. Additionally, we demonstrated that mitochondrial fusion was enhanced in diabetic hearts. Therefore, direct stimulation of mTOR by Leu might be effective in cardiac preconditioning in a diabetic model.

\section{Conclusion}

Leu treatment resulted in cardiac preconditioning with increased mTOR activity and mitochondrial fusion in both wild-type and HFD-induced obese mice, thereby suggesting that Leu could be potentially used for myocardial I/R injury treatment in patients with diabetes.

\section{List Of Abbreviations}

CAD: coronary artery disease

AMl: acute myocardial infarction

I/R: ischemia/reperfusion

BCAA: branched-chain amino acid

mTOR: mammalian target of rapamycin

Leu: leucine

HFD: high-fat diet 
LAD: left coronary artery

IPC: ischemic preconditioning

ROS: reactive Oxygen Species

Rap: rapamycin

PBS: phosphate-buffered saline

EM: electron microscope

\section{Declarations}

\subsection{Ethics approval and consent to participate}

The protocols were approved by the Animal Care and Use Committee at the University of Tokushima.

\subsection{Consent for publication}

Not applicable

\subsection{Availability of data and materials}

The datasets used and analyzed during the current study are available from the corresponding author on reasonable request.

\subsection{Competing interests}

The authors declare that they have no competing interests.

\subsection{Funding}

This work was supported by JSPS KAKENHI, Japan [grant number 19K09353].

\subsection{Authors' contributions}

AM and RT conceived and carried out the experiments. AM and SS wrote the manuscript with support from RT, TK, KH, and NS. TK, HM, TK, and MK contributed to the sample preparation and perform analytic calculations. HS and YT helped supervise the project.

\subsection{Acknowledgements}

Not applicable

\section{References}


1. J.A. Beckman, M.A. Creager, P. Libby, Diabetes and atherosclerosis: epidemiology, pathophysiology, and management, Jama 287 (2002) 2570-2581. 10.1001/jama.287.19.2570.

2. M. Ding, J. Lei, H. Han, W. Li, Y. Qu, E. Fu, F. Fu, X. Wang, SIRT1 protects against myocardial ischemiareperfusion injury via activating eNOS in diabetic rats, Cardiovasc Diabetol 14 (2015) 143. 10.1186/s12933-015-0299-8.

3. K. Tanaka, F. Kehl, W. Gu, J.G. Krolikowski, P.S. Pagel, D.C. Warltier, J.R. Kersten, Isoflurane-induced preconditioning is attenuated by diabetes, Am J Physiol Heart Circ Physiol 282 (2002) H2018-2023. 10.1152/ajpheart.01130.2001.

4. D. Aguilar, S.D. Solomon, L. Køber, J.L. Rouleau, H. Skali, J.J. McMurray, G.S. Francis, M. Henis, C.M. O'Connor, R. Diaz, Y.N. Belenkov, S. Varshavsky, J.D. Leimberger, E.J. Velazquez, R.M. Califf, M.A. Pfeffer, Newly diagnosed and previously known diabetes mellitus and 1-year outcomes of acute myocardial infarction: the VALsartan In Acute myocardial iNfarcTion (VALIANT) trial, Circulation 110 (2004) 1572-1578. 10.1161/01.Cir.0000142047.28024.F2.

5. R.J. Stevens, R.L. Coleman, A.I. Adler, I.M. Stratton, D.R. Matthews, R.R. Holman, Risk factors for myocardial infarction case fatality and stroke case fatality in type 2 diabetes: UKPDS 66, Diabetes Care 27 (2004) 201-207. 10.2337/diacare.27.1.201.

6. H. Li, Z. Liu, J. Wang, G.T. Wong, C.W. Cheung, L. Zhang, C. Chen, Z. Xia, M.G. Irwin, Susceptibility to myocardial ischemia reperfusion injury at early stage of type 1 diabetes in rats, Cardiovasc Diabetol 12 (2013) 133. 10.1186/1475-2840-12-133.

7. H.H. Patel, Y.M. Tsutsumi, B.P. Head, I.R. Niesman, M. Jennings, Y. Horikawa, D. Huang, A.L. Moreno, P.M. Patel, P.A. Insel, D.M. Roth, Mechanisms of cardiac protection from ischemia/reperfusion injury: a role for caveolae and caveolin-1, FASEB J 21 (2007) 1565-1574. 10.1096/fj.06-7719com.

8. Y.M. Tsutsumi, T. Yokoyama, Y. Horikawa, D.M. Roth, H.H. Patel, Reactive oxygen species trigger ischemic and pharmacological postconditioning: in vivo and in vitro characterization, Life Sci 81 (2007) 1223-1227. 10.1016/j.lfs.2007.08.031.

9. B. Ibáñez, G. Heusch, M. Ovize, F. Van de Werf, Evolving therapies for myocardial ischemia/reperfusion injury, J Am Coll Cardiol 65 (2015) 1454-1471. 10.1016/j.jacc.2015.02.032.

10. Y.M. Tsutsumi, Y. Kawaraguchi, I.R. Niesman, H.H. Patel, D.M. Roth, Opioid-induced preconditioning is dependent on caveolin-3 expression, Anesth Analg 111 (2010) 1117-1121.

10.1213/ANE.0b013e3181f3351a.

11. C.M. Stary, Y.M. Tsutsumi, P.M. Patel, B.P. Head, H.H. Patel, D.M. Roth, Caveolins: targeting prosurvival signaling in the heart and brain, Front Physiol 3 (2012) 393. 10.3389/fphys.2012.00393.

12. S. Satomi, A. Morio, H. Miyoshi, R. Nakamura, R. Tsutsumi, H. Sakaue, T. Yasuda, N. Saeki, Y.M. Tsutsumi, Branched-chain amino acids-induced cardiac protection against ischemia/reperfusion injury, Life Sci 245 (2020) 117368. 10.1016/j.lfs.2020.117368.

13. C. Sanchez Canedo, B. Demeulder, A. Ginion, J.R. Bayascas, J.L. Balligand, D.R. Alessi, J.L. Vanoverschelde, C. Beauloye, L. Hue, L. Bertrand, Activation of the cardiac mTOR/p70(S6K) pathway 
by leucine requires PDK1 and correlates with PRAS40 phosphorylation, Am J Physiol Endocrinol Metab 298 (2010) E761-769. 10.1152/ajpendo.00421.2009.

14. C. Kilkenny, W.J. Browne, I.C. Cuthill, M. Emerson, D.G. Altman, Improving bioscience research reporting: the ARRIVE guidelines for reporting animal research, PLoS Biol 8 (2010) e1000412. 10.1371/journal.pbio.1000412.

15. M. Murakami, T. Ichisaka, M. Maeda, N. Oshiro, K. Hara, F. Edenhofer, H. Kiyama, K. Yonezawa, S. Yamanaka, mTOR is essential for growth and proliferation in early mouse embryos and embryonic stem cells, Mol Cell Biol 24 (2004) 6710-6718. 10.1128/mcb.24.15.6710-6718.2004.

16. Y.M. Tsutsumi, Y.T. Horikawa, M.M. Jennings, M.W. Kidd, I.R. Niesman, U. Yokoyama, B.P. Head, Y. Hagiwara, Y. Ishikawa, A. Miyanohara, P.M. Patel, P.A. Insel, H.H. Patel, D.M. Roth, Cardiac-specific overexpression of caveolin-3 induces endogenous cardiac protection by mimicking ischemic preconditioning, Circulation 118 (2008) 1979-1988. 10.1161/circulationaha.108.788331.

17. Y.T. Horikawa, H.H. Patel, Y.M. Tsutsumi, M.M. Jennings, M.W. Kidd, Y. Hagiwara, Y. Ishikawa, P.A. Insel, D.M. Roth, Caveolin-3 expression and caveolae are required for isoflurane-induced cardiac protection from hypoxia and ischemia/reperfusion injury, J Mol Cell Cardiol 44 (2008) 123-130. 10.1016/j.yjmcc.2007.10.003.

18. K. Hirose, Y.M. Tsutsumi, R. Tsutsumi, M. Shono, E. Katayama, M. Kinoshita, K. Tanaka, S. Oshita, Role of the O-linked $\beta$ - $\mathrm{N}$-acetylglucosamine in the cardioprotection induced by isoflurane, Anesthesiology 115 (2011) 955-962. 10.1097/ALN.0b013e31822fcede.

19. Y.M. Tsutsumi, R. Tsutsumi, E. Hamaguchi, Y. Sakai, A. Kasai, Y. Ishikawa, U. Yokoyama, K. Tanaka, Exendin-4 ameliorates cardiac ischemia/reperfusion injury via caveolae and caveolins-3, Cardiovasc Diabetol 13 (2014) 132. 10.1186/s12933-014-0132-9.

20. Y.M. Tsutsumi, Y. Kawaraguchi, Y.T. Horikawa, I.R. Niesman, M.W. Kidd, B. Chin-Lee, B.P. Head, P.M. Patel, D.M. Roth, H.H. Patel, Role of caveolin-3 and glucose transporter-4 in isoflurane-induced delayed cardiac protection, Anesthesiology 112 (2010) 1136-1145. 10.1097/ALN.0b013e3181d3d624.

21. Y.T. Horikawa, M. Panneerselvam, Y. Kawaraguchi, Y.M. Tsutsumi, S.S. Ali, R.C. Balijepalli, F. Murray, B.P. Head, I.R. Niesman, T. Rieg, V. Vallon, P.A. Insel, H.H. Patel, D.M. Roth, Cardiac-specific overexpression of caveolin-3 attenuates cardiac hypertrophy and increases natriuretic peptide expression and signaling, J Am Coll Cardiol 57 (2011) 2273-2283. 10.1016/j.jacc.2010.12.032.

22. R. Matsushima, A. Takahashi, Y. Nakaya, H. Maezawa, M. Miki, Y. Nakamura, F. Ohgushi, S. Yasuoka, Human airway trypsin-like protease stimulates human bronchial fibroblast proliferation in a proteaseactivated receptor-2-dependent pathway, Am J Physiol Lung Cell Mol Physiol 290 (2006) L385-395. 10.1152/ajplung.00098.2005.

23. V. Parra, V. Eisner, M. Chiong, A. Criollo, F. Moraga, A. Garcia, S. Härtel, E. Jaimovich, A. Zorzano, C. Hidalgo, S. Lavandero, Changes in mitochondrial dynamics during ceramide-induced cardiomyocyte early apoptosis, Cardiovasc Res 77 (2008) 387-397. 10.1093/cvr/cvm029. 
24. T. Yu, J.L. Robotham, Y. Yoon, Increased production of reactive oxygen species in hyperglycemic conditions requires dynamic change of mitochondrial morphology, Proc Natl Acad Sci U S A 103 (2006) 2653-2658. 10.1073/pnas.0511154103.

25. Y.M. Tsutsumi, H.H. Patel, N.C. Lai, T. Takahashi, B.P. Head, D.M. Roth, Isoflurane produces sustained cardiac protection after ischemia-reperfusion injury in mice, Anesthesiology 104 (2006) 495-502. 10.1097/00000542-200603000-00017.

26. C.G. Proud, Regulation of mammalian translation factors by nutrients, European Journal of Biochemistry 269 (2002) 5338-5349. https://doi.org/10.1046/j.1432-1033.2002.03292.x.

27. L. Tilokani, S. Nagashima, V. Paupe, J. Prudent, Mitochondrial dynamics: overview of molecular mechanisms, Essays Biochem 62 (2018) 341-360. 10.1042/ebc20170104.

28. S. Nagdas, D.F. Kashatus, The Interplay between Oncogenic Signaling Networks and Mitochondrial Dynamics, Antioxidants 6 (2017) 33.

29. H. Kalkavan, D.R. Green, MOMP, cell suicide as a BCL-2 family business, Cell Death Differ 25 (2018) 46-55. 10.1038/cdd.2017.179.

30. K.G. de la Cruz López, M.E. Toledo Guzmán, E.O. Sánchez, A. García Carrancá, mTORC1 as a Regulator of Mitochondrial Functions and a Therapeutic Target in Cancer, Frontiers in Oncology 9 (2019). 10.3389/fonc.2019.01373.

31. M. Morita, J. Prudent, K. Basu, V. Goyon, S. Katsumura, L. Hulea, D. Pearl, N. Siddiqui, S. Strack, S. McGuirk, J. St-Pierre, O. Larsson, I. Topisirovic, H. Vali, H.M. McBride, J.J. Bergeron, N. Sonenberg, mTOR Controls Mitochondrial Dynamics and Cell Survival via MTFP1, Mol Cell 67 (2017) 922935.e925. 10.1016/j.molcel.2017.08.013.

32. A. Szabo, K. Sumegi, K. Fekete, E. Hocsak, B. Debreceni, G. Setalo, Jr., K. Kovacs, L. Deres, A. Kengyel, D. Kovacs, J. Mandl, M. Nyitrai, M.A. Febbraio, F. Gallyas, Jr., B. Sumegi, Activation of mitochondrial fusion provides a new treatment for mitochondria-related diseases, Biochem Pharmacol 150 (2018) 86-96. 10.1016/j.bcp.2018.01.038.

33. P.P. Devarshi, S.M. McNabney, T.M. Henagan, Skeletal Muscle Nucleo-Mitochondrial Crosstalk in Obesity and Type 2 Diabetes, Int J Mol Sci 18 (2017). 10.3390/ijms18040831.

34. J. Russell, E.F. Du Toit, J.N. Peart, H.H. Patel, J.P. Headrick, Myocyte membrane and microdomain modifications in diabetes: determinants of ischemic tolerance and cardioprotection, Cardiovasc Diabetol 16 (2017) 155. 10.1186/s12933-017-0638-z.

35. R. Engbersen, N.P. Riksen, M.J. Mol, B. Bravenboer, O.C. Boerman, P. Meijer, W.J. Oyen, C. Tack, G.A. Rongen, P. Smits, Improved resistance to ischemia and reperfusion, but impaired protection by ischemic preconditioning in patients with type 1 diabetes mellitus: a pilot study, Cardiovasc Diabetol 11 (2012) 124. 10.1186/1475-2840-11-124.

36. M.H. Stipanuk, Leucine and protein synthesis: mTOR and beyond, Nutr Rev 65 (2007) 122-129. 10.1111/j.1753-4887.2007.tb00289.x.

\section{Figures}


Figure 1

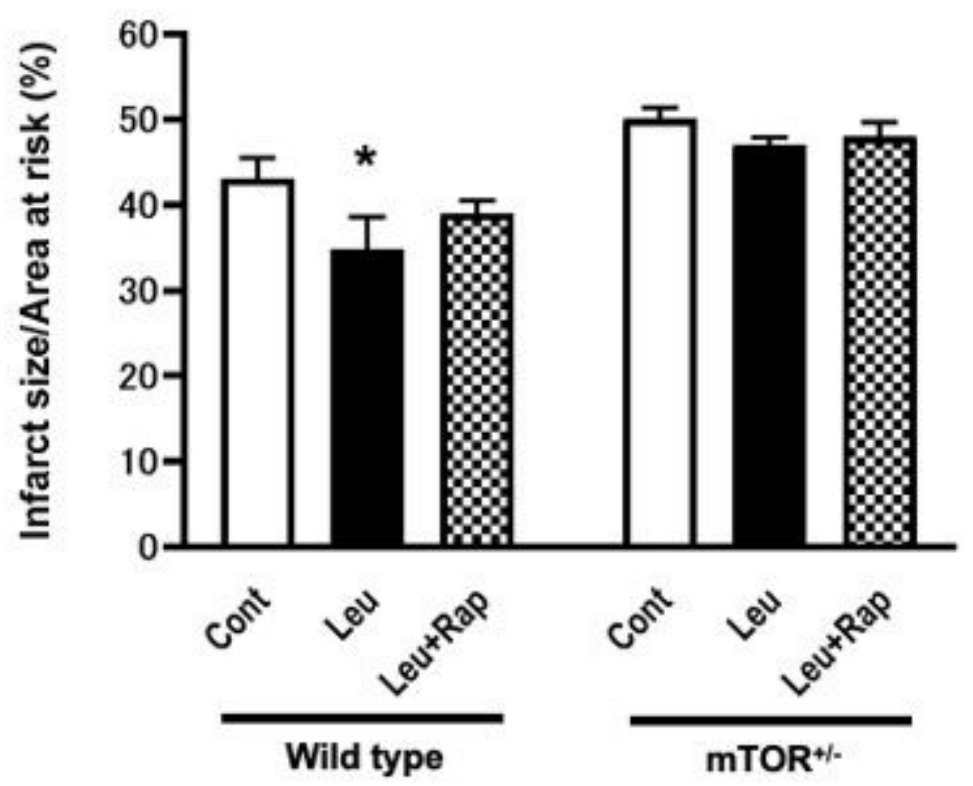

Figure 1

Effects of leucine (Leu) on myocardial ischemia/reperfusion (I/R) injury in mice Leu reduced infarct size after I/R injury in wild-type mice. The protective effect of Leu was attenuated by rapamycin (Rap). Leu did not improve the infarct size in mTOR $+/-$ mice ${ }^{*} p<0.05$, vs. control (Cont). mTOR, mammalian target of rapamycin. 
Figure 2
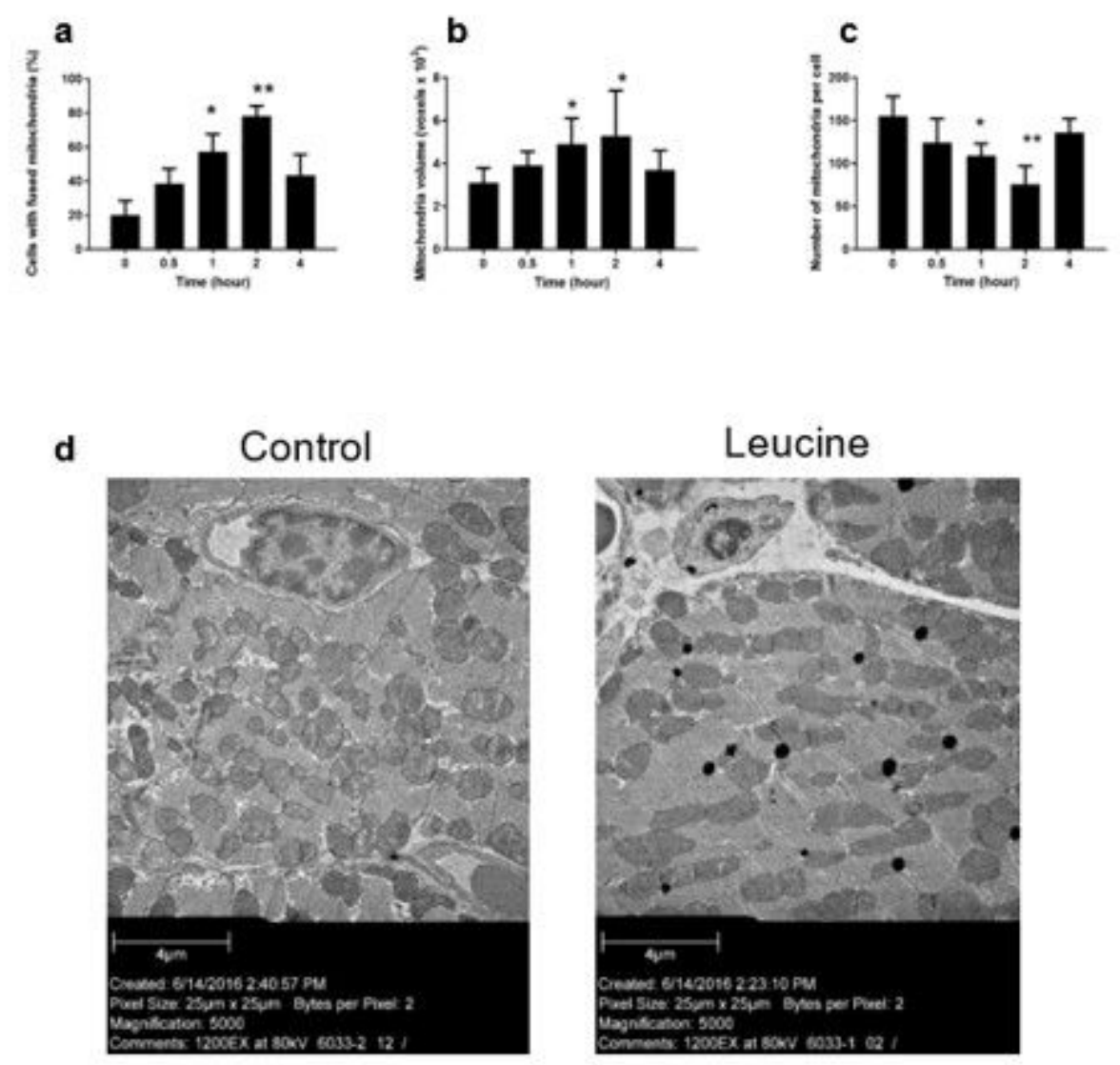

\section{Figure 2}

Effects of leucine (Leu) on mitochondria fusion in cardiomyocytes The percentage of cells displaying (a) fused mitochondria and the (b) mitochondrial volume and (c) the number of mitochondria per cell were evaluated for $4 \mathrm{~h}$ at $30 \mathrm{~min}$ intervals after Leu pretreatment on isolated rat cardiomyocytes. The result of time 0 was used as the baseline. Leu led to an increase in mitochondrial fusion and mitochondrial volume and a decrease in the number of mitochondria after $2 \mathrm{~h}$ of incubation. ${ }^{*} p<0.05,{ }^{*} \mathrm{p}<0.01$ vs. control (Cont). (d) Leu treatment $(160 \mu \mathrm{M})$ for $2 \mathrm{~h}$ caused a decrease in the number of mitochondria and an increase in mitochondrial fusion in cardiomyocytes. 
Figure3
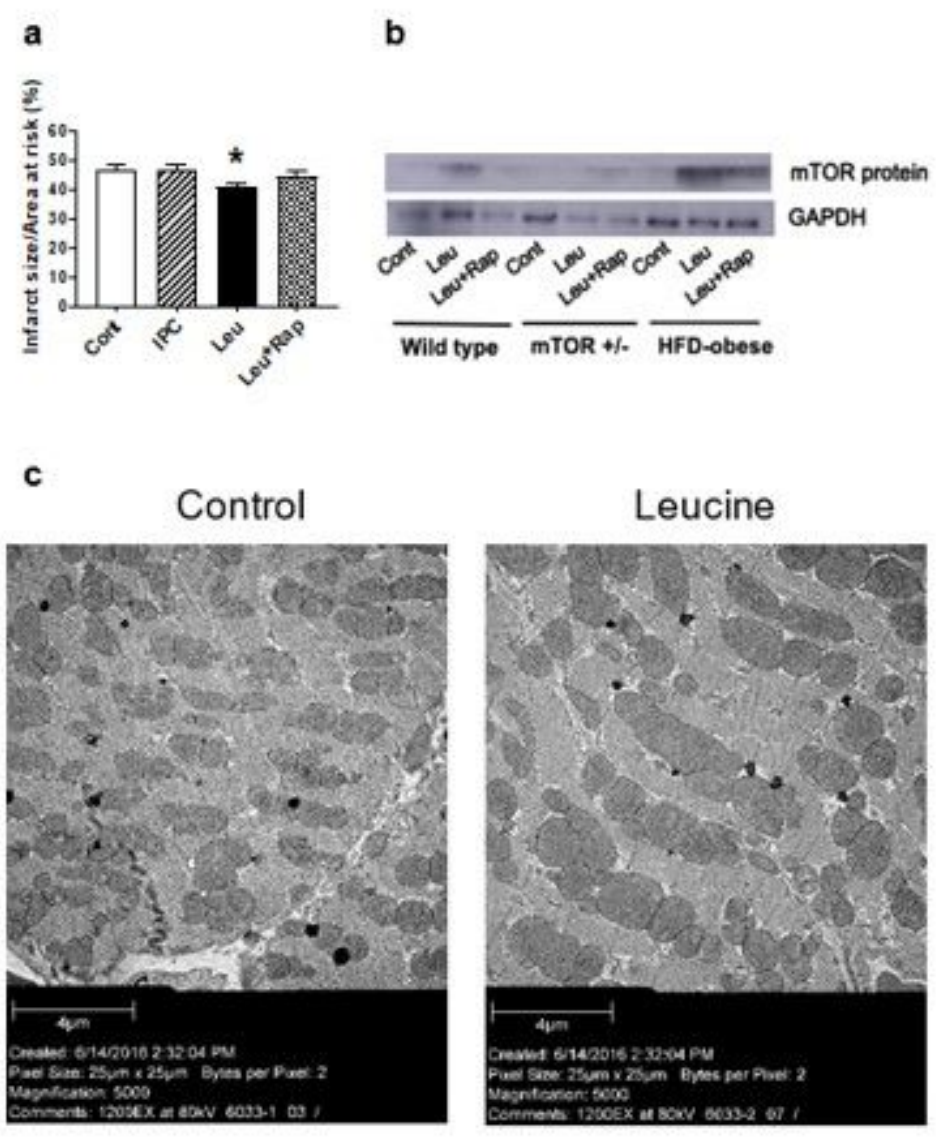

\section{Figure 3}

Effects of leucine (Leu) on ischemia/reperfusion (I/R) injury and mitochondrial fusion in high-fat diet (HFD)-induced obese mice (a) HFD-induced obese mice were subjected to I/R. No significant difference in infarct size between the control (Cont) group and the ischemic preconditioning (IPC) group was observed, whereas Leu treatment reduced the infarct size caused by I/R injury in HFD-induced obese mice. The protective effect of Leu was abrogated by rapamycin (Rap). * $p<0.05$, vs. Cont. (b) mTOR protein expression was examined in wild-type, mTOR+/-, and HFD-induced obese mice. Leu treatment increased mTOR expression in wild-type and HFD-induced obese mice, but not in mTOR+/- mice. Leu, leucine; HFD, high-fat diet; mTOR, mammalian target of rapamycin; Rap, rapamycin; Cont, control; GAPDH, glyceraldehyde 3-phosphate dehydrogenase. (c) The changes in mitochondrial dynamics caused by Leu were determined using electron microscopy. Enhanced mitochondrial fusion was observed in the heart tissues of HFD-induced obese mice. 


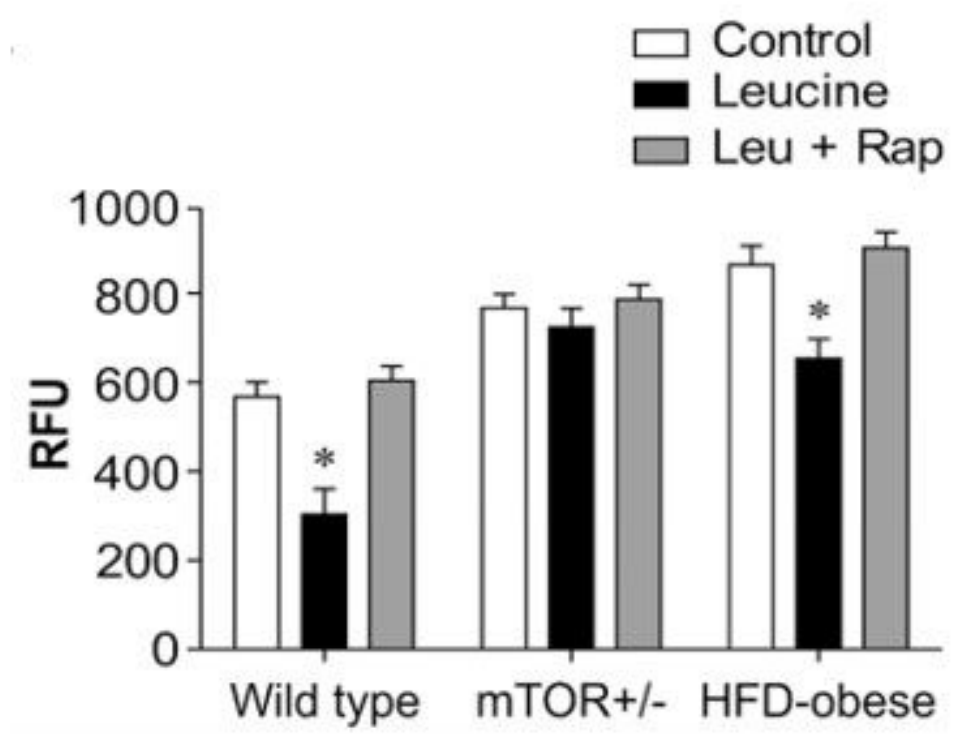

Figure 4

Reactive oxygen species (ROS) production in myocardium pretreated with leucine (Leu) ROS generation was examined after inducing ischemia/reperfusion injury in wild-type, mTOR+/-, and HFD-induced obese mice. Leu treatment resulted in decreased ROS generation in wild-type and HFD-induced obese mice, and this effect was inhibited by Rap. ${ }^{*} p<0.05$, vs. control (Cont). mTOR, mammalian target of rapamycin; Rap, rapamycin; RFU, relative fluorescence units. 